\title{
A nested square-shape dielectric resonator for microwave band antenna applications
}

\author{
Ubaid Ullah', Ismail Ben Mabrouk $^{2}$, Muath Al-Hasan ${ }^{3}$, Mourad Nedil ${ }^{4}$, Mohd Fadzil Ain ${ }^{5}$ \\ $1,2,3$ Networks and Communication Engineering Department, Al Ain University, United Arab Emirates \\ ${ }^{4}$ Engineering Department, Université de Québec, Canada \\ ${ }^{5}$ School of Electrical and Electronic Engineering, Universiti Sains Malaysia, Malaysia
}

\section{Article Info}

Article history:

Received Jan 2, 2020

Revised Jun 30, 2020

Accepted Jul 13, 2020

\section{Keywords:}

Dielectric resonator antenna

Microwave band

Nested square-shape dielectric

Square-shape dielectric

Wideband antenna

\begin{abstract}
In this paper, a nested square-shape dielectric resonator (NSDR) has been designed and investigated for antenna applications in the microwave band. A solid square dielectric resonator (SSDR) was modified systematically by introducing air-gap in the azimuth ( $\phi$-direction). By retaining the square shape of the dielectric resonator (DR), the well-known analysis tools can be applied to evaluate the performance of the NSDR. To validate the performance of the proposed NSDR in antenna applications, theoretical, simulation, and experimental analysis of the subject has been performed. A simple microstrip-line feeding source printed on the top of Rogers RO4003 grounded substrate was utilized without any external matching network. Unlike solid square DR, the proposed NSDR considerably improves the impedance bandwidth. The proposed antenna has been prototyped and experimentally validated. The antenna operates in the range of $12.34 \mathrm{GHz}$ to $21.7 \mathrm{GHz}$ which corresponds to $56 \%$ percentage bandwidth with peak realized gain $6.5 \mathrm{~dB}$. The antenna has stable radiation characteristics in the broadside direction. A close agreement between simulation and experimental results confirms the improved performance of NSDR in antenna applications.
\end{abstract}

This is an open access article under the CC BY-SA license.

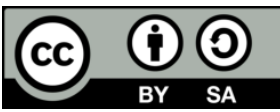

Corresponding Author:

Ubaid Ullah,

Networks and Communication Engineering Department,

Al Ain University,

Abu Dhabi 112612, United Arab Emirates.

Email: ubaid.ullah@aau.ac.ae

\section{INTRODUCTION}

Dielectric resonator antennas (DRAs) belong to the most promising types of antenna structures researched over the recent years. This is not only because of their attractive physical characteristics (low profile, small size, ease of excitation, and fabrication) but also their good performance at high frequencies [1]. The rich literature on DRAs shows that so far they are the best alternatives to the conventionally used metallic patch antennas which are generally criticized for their intrinsic narrowband operation $[2,3]$. In contemporary communication systems, the antenna size is an important design consideration. DRA offers greater flexibility with this respect as its size scales down by a factor of $\varepsilon_{r}^{-1 / 2}$, which is better than for its metallic counterparts. In particular, a DRA can be made ten times smaller by scaling the permittivity from 10 to 100 . Another important feature of DRAs is the potential for achieving a wider impedance bandwidth, which is due to the direct dependence of the impedance bandwidth on the permittivity of the resonator [4-6]. 
To meet the demands of modern communication systems, it is necessary to reduce the size of the antenna while maintaining a wide impedance bandwidth as well as stable radiation characteristics [7-11]. With high permittivity material, a small size along with a high radiation efficiency can be achieved only for a very narrow operating band [12-14]. Utilization of medium values of dielectric constants facilitates the realization of a required impedance bandwidth and a small area allocated for the antenna footprint in the communication systems but it is usually insufficient to fulfill all the design requirements [15-17]. In order to design a dielectric resonator antenna that can operate with stable radiation properties, it is required to excite a particular mode of the dielectric resonator that meets the radiation characteristics of the intended application. For the standard shapes of the DRA (cylindrical, rectangular, square) the in-depth analysis has already been done by many researchers. In particular, the fundamental modes are the preferred choices for electric and magnetic dipole-like radiation characteristics [18-23]. Given the rich literature and engineering knowledge on almost every aspect of homogeneous dielectric resonators, it is possible to explore the characteristics of inhomogeneous dielectric resonators [24].

To date, very little research has been published in which the dielectric resonator physical shape and geometry is maintained while the permittivity is altered [25, 26]. In this work, novel inhomogeneous nested square-shape dielectric resonators (NSDR) are proposed, in which inhomogeneity (air-gap) will be introduced in the azimuth $(\phi)$ direction so that the original shape of the resonator and its radiating characteristics remain uninterrupted. The systematic design of NSDR involving theoretical, simulation, and approximate analysis, is followed by its application for antenna design. The contribution in this paper includes: (i) development of a new inhomogeneous nested-square shape dielectric resonator; (ii) theoretical and numerical analysis of the new dielectric resonator; (iii) implementation and validation of the newly developed resonator for antenna applications in the microwave band.

\section{NESTED-SQUARE SHAPE DIELECTRIC RESONATOR}

In this section, the design and configuration of a new inhomogeneous NSDR is presented. Square shape dielectric resonator was chosen for this design with the intent to use it for circularly polarized antennas due to its flexibility in exciting orthogonal modes. The idea behind this design is the fact that when a dielectric material of a relatively high permittivity is placed in an electric field, the density of the electric energy will increase in the surrounding of the dielectric material and it will decrease inside the high permittivity material. In case there is a direct contact between a conductor and a dielectric material (i.e. direct microstrip-line-fed dielectric resonator), the intensity of the electric field increases at the point of contact between the conductor and the resonator, in its immediate vicinity, and inside the resonator. Theoretically, the field strength may also increase to infinity depending on the type of connection between the conductor and the resonator as well as permittivity of the dielectric material placed on the top of the conductor [16]. Keeping this in mind, a new inhomogeneous nested square-shape dielectric resonator is designed and analyzed for wideband applications.

The E-field pattern present in a solid square shape dielectric resonator and a square ring dielectric resonator is depicted in Figure 1. This indicates that by altering the geometry of the square DR, the electrical length of the resonator is increased which leads to enhancing the impedance bandwidth. The phenomenon of lengthening the electrical current path is also utilized to achieve a low profile structure that can be operated within a wide frequency range. A rigorous time-domain analysis of the resonator revealed that the bandwidth of each mode can be increased by altering the geometry of the resonator in such a way that the electromagnetic field of the fundamental mode remains the same. By properly optimizing the geometry of the square shape DR, the constructive interference of the electromagnetic fields inside the resonator for the desired mode can be increased which eventually leads to achieving a wideband response of the antenna.

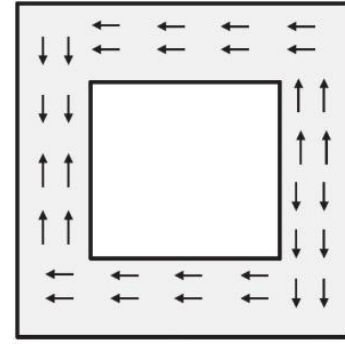

$\mathbf{a}$

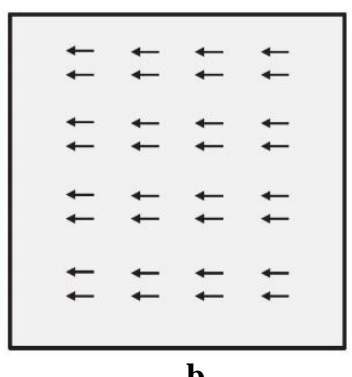

$\mathbf{b}$

Figure 1. Orientation of electric field, (a) Square ring dielectric resonator (b) Solid square dielectric resonator 


\section{ANALYTICAL AND NUMERICAL ANALYSIS}

For the analytical solution of the proposed inhomogeneous NSDR, the dielectric waveguide model (DWM) is employed. The overall geometry of the inhomogeneous dielectric resonator was maintained similar to a solid square dielectric resonator (SSDR) to easily investigate and compare the mode patterns of the proposed NSDR. The resonant frequency of the $\mathrm{TE}_{\mathrm{mnl}}$ mode can be predicted using the DWM equations give below in its simplest form applied to a solid rectangular DR.

$$
f_{o}=\frac{c}{2 \pi \sqrt{\varepsilon_{r}}} \sqrt{K_{x}^{2}+K_{y}^{2}+K_{z}^{2}}
$$

where

$$
K_{x}=\frac{m \pi}{a}, K_{y}=\frac{n \pi}{b}, K_{z}=\frac{l \pi}{2 d}
$$

and

$$
K_{x}^{2}+K_{y}^{2}+K_{z}^{2}=\varepsilon_{r} K_{o}^{2}
$$

The mode patterns for the baseline design of a solid resonator having the same permittivity as that of the proposed DR have been visualized in Figure 2. A detailed discussion on the E-field and H-field patterns of the solid resonator based on the frequency domain analysis and the field distributions for the first three modes supported by a rectangular DR can be found in [11]. From this electromagnetic field analysis of a solid rectangular dielectric resonator and the theory mentioned in the previous section, it is assumed that the performance of the rectangular DR can be improved with proper alteration of its geometrical configuration. The isolated geometry of the proposed inhomogeneous nested square-shape DR is shown in Figure 3. The dimensions of the outer square ring are kept to $1.5 \mathrm{~mm}$ while inhomogeneity in the form of $0.5 \mathrm{~mm}$ air was introduced followed by another square ring of $1 \mathrm{~mm}$ and the innermost square of $7 \mathrm{~mm}$ was kept solid to maintain electromagnetic energy field pattern in the core of the square DR.

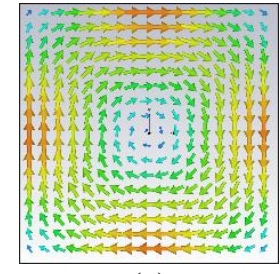

(a)

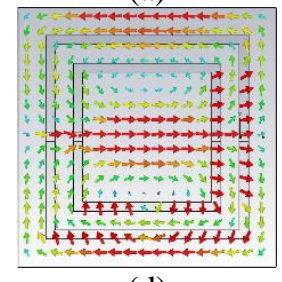

(d)

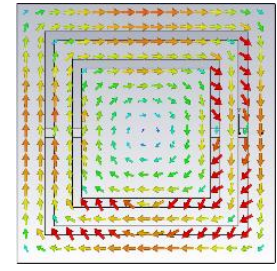

(b)

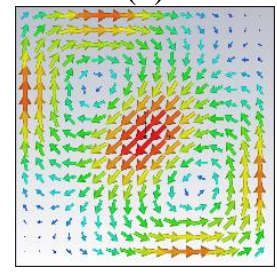

(e)

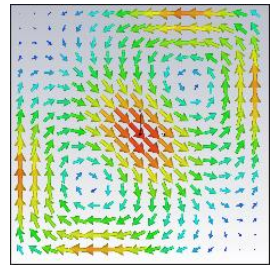

(c)

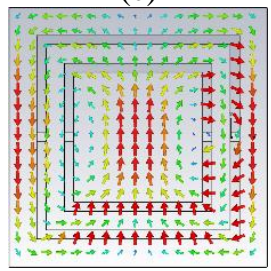

(f)

Figure 2. Electric field distribution in $x-y$ direction of a solid square (SS) and nested square (NS) dielectric resonator, (a) $\mathrm{TE}_{11}$ E-field in SS, (b) $\mathrm{TE}_{11}$ E-field in NS, (c) $\mathrm{TE}_{12}$ E-field in SS, (d) $\mathrm{TE}_{12}$ E-field in NS,

(e) $\mathrm{TE}_{12 \mathrm{~d}}$ E-field in SS, (f) $\mathrm{TE}_{12 \mathrm{~d}}$ E-field in NS

To achieve the best possible performance in terms of the impedance bandwidth of the antenna, dimensions of the NSDR are optimized through parametric analysis. Once a wideband response has been achieved, an in-depth analysis of the electromagnetic field distribution of an isolated SSDR and NSDR was performed using a finite-difference eigenmode (FDE) solver. The E-field and H-field values of the first three modes are illustrated and discussed to understand the wideband behavior of the proposed NSDR in 
comparison with its solid counterpart. The electromagnetic field distribution in the SSDR is different from the fields in the NSDR which is highlighted in terms of the E-field of the first three modes. Figures 2(a) and 2(b) depict the E-field for the first resonant mode $\mathrm{TE}_{11}$ in $\mathrm{x}-\mathrm{y}$ direction of a solid square (SS) and nested square (NS) dielectric resonators, respectively.

The arrows indicate a different pattern of the E-field for SS and NS which is mainly because of the presence of air-gap introduced in the NSDR. Figures 2(c) and 2(d) show the TE 12 mode while the same mode has degenerated with a 90-degree phase-shifted field as illustrated in Figure 2(e) and 2(f). This mode is represented as $\mathrm{TE}_{12 \mathrm{~d}}$, whereas $d$ in the subscript refers to the degenerate nature of the mode. Maximum energy can be coupled to the dielectric resonator when the external feeding source is placed in the area where the electric (Js)/magnetic (Ms) fields are at their peak. For determining the exact amount of coupling from the source to the resonator, the reciprocity theorem can be applied with proper boundary conditions. As can be seen from the illustrated fields in the $x-y$ direction of the first three modes in the proposed NSDR, the maximum energy is confined within the diagonal of the resonator. Hence, placing the external feeding source at the diagonal will result in maximum coupling to the resonator.

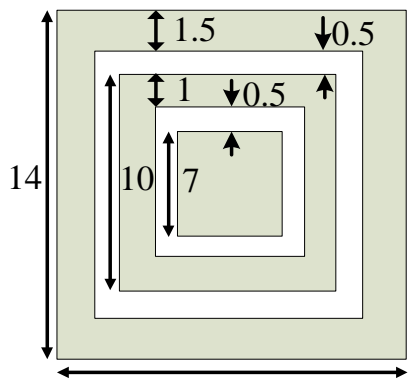

Figure 3. Geometry and prototype of the proposed nested square shape DR

\section{NSDR ANTENNA DEVELOPMENT}

To confirm the wideband response of the NSDR in antenna applications, simulation, and experimental analysis has been performed. For time-domain analysis, CST Microwave Studio was used whereas for experimental characterization roger RT6010 $\left(\varepsilon_{r}=10.2\right)$ with thickness $t=8 \mathrm{~mm}$ was utilized for NSDR due to its flexibility and easy mechanical processing. For antenna applications, the NSDR was loaded on top of the grounded Rogers RO4003 substrate $\left(\varepsilon_{r}=3.38\right)$. The antenna has been developed in four stages as depicted in Figure 4(a). The impedance bandwidth is analyzed with each stage as shown in Figure 4(b). A simple microstrip line feeding technique is employed for the excitation of NSDR by placing it diagonally with respect to the T-junction at the open end of the microstrip line. For the first stage, a resonance near $20.5 \mathrm{GHz}$ can be observed but the impedance matching is not sufficient. By adding the first square-ring in the second stage of the design, an impedance matching below - 10dB is achieved covering the frequency range from $17.8 \mathrm{GHz}$ to $22 \mathrm{GHz}$. For the third design stage, a wide impedance bandwidth from $12.9 \mathrm{GHz}$ to almost $22 \mathrm{GHz}$. By adding an additional ring to the resonator in the fourth stage the starting frequency is lowered from $12.9 \mathrm{GHz}$ to $12.4 \mathrm{GHz}$ but the impedance matching is compromised. It is imperative to mention here that until this point the lateral position of the DR with respect to the feedline has not been optimized.
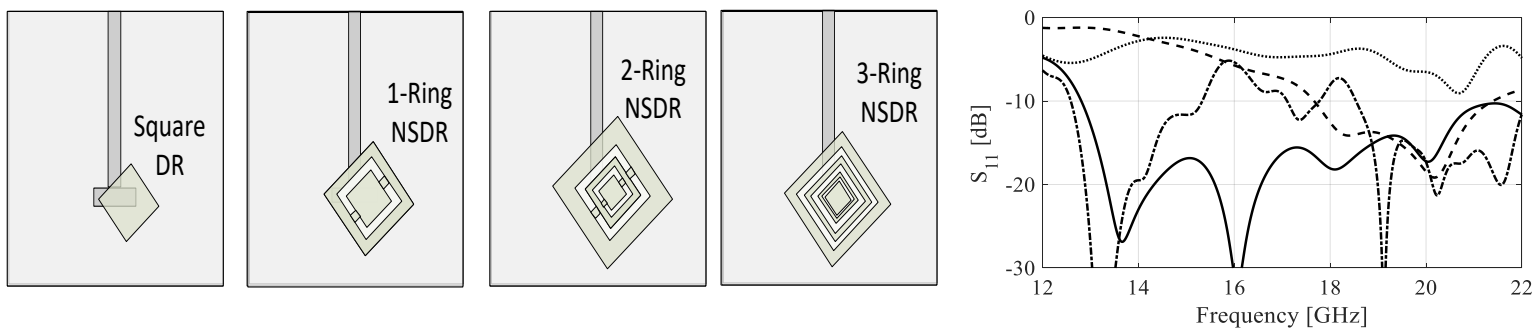

(a)

(b)

Figure 4. Antenna analysis through different nest size, (a) Development stages, (b) Impedance matching 
Due to fabrication limitation the NSDR with two number of rings has been selected and tuned for final prototyping. By properly adjusting the orientation of the NSDR at the top of the microstrip line, transverse electric (TE) modes can be efficiently excited in the DR with broader bandwidth due to the inhomogeneity introduced in the square dielectric resonator. The simulated and prototype profile of the nested square shape DRA is shown in Figure 5.

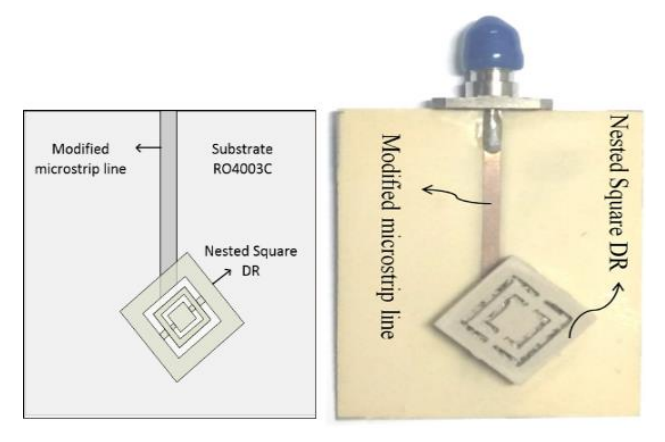

Figure 5. Simulation and prototype of the proposed NSDRA

\section{RESULTS AND DISCUSSION}

\subsection{Impedance bandwidth}

Provide the impedance bandwidth characterization of the proposed inhomogeneous NSDRA is implemented through the time domain simulation at the full-wave EM level of the description using CST microwave studio. For the experimental characterization and validation, the impedance bandwidth measurements are performed using the Agilent Technology PNA-XN6245A network analyzer. The simulated and measured scattering parameter $|\mathrm{S} 11|<-10 \mathrm{~dB}$ is illustrated in Figure 6. The impedance bandwidth of the antenna is $12.34 \mathrm{GHz}$ to $21.72 \mathrm{GHz}$ (56 percent) with a close agreement between simulation and measurement. In comparison to the simulated impedance bandwidth of NSDRA, a $130 \mathrm{MHz}$ upward shift in the measured frequency is observed. These slight changes can be attributed to human error, imperfect experimental environment, and mechanical stability of the antenna.

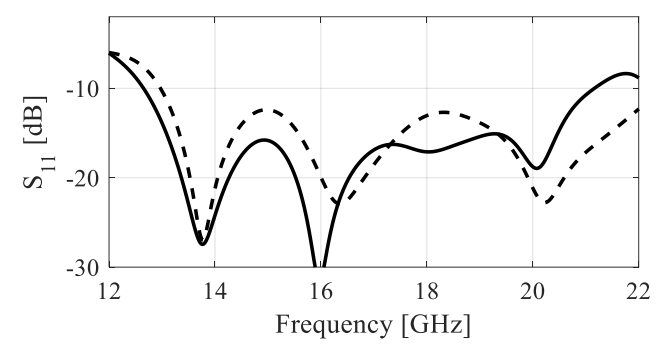

Figure 6. Impedance bandwidth response of NSDRA, simulated (- - -), measured ( - )

\subsection{Radiation pattern characterization of NSDRA}

Radiation pattern characteristics of inhomogeneous nested square dielectric resonator antenna is evaluated at three different frequencies within the operating bandwidth of the antenna. The co-polarized (Co-pol) and cross-polarized (X-pol) E-plane and H-plane magnitudes and phase values at the main lobe direction are analyzed. Similar to the previous designs, three different frequency points are evaluated near the low-end frequency of $13.5 \mathrm{GHz}$, center frequency $17 \mathrm{GHz}$, and near high-end frequency of $21 \mathrm{GHz}$ in the operating impedance bandwidth of the proposed antenna.

The simulated and measured E-plane and H-plane at $13.5 \mathrm{GHz}$ which is close to the lower end of the operating bandwidth of the antenna is given in Figure 7(a) and 7(b) for both X-pol and Co-pol planes. The maximum magnitude and phase value achieved for the Co-pol main lobe of the E-plane were found to be $8.09 \mathrm{~dB}$ at $0^{\circ}$ and $8.15 \mathrm{~dB}$ at $0^{\circ}$ for simulation and measurement respectively. For $\mathrm{X}$-pol, the highest magnitude found was $-1.11 \mathrm{~dB}$ at $30^{\circ}$ for simulation and -0.594 at $-80^{\circ}$ for measurement as shown in Figure 7(a). Similarly, the maximum magnitude of the Co-pol H-plane at $13.5 \mathrm{GHz}$ for simulation and

A nested square-shape dielectric resonator for microwave band antenna applications (Ubaid Ullah) 
measurement was recorded as $7.9 \mathrm{~dB}$ and $8.05 \mathrm{~dB}$ both oriented at $0^{\circ}$ while the highest X-pole magnitudes were $-2.49 \mathrm{~dB}$ at $-69^{\circ}$ and $0.439 \mathrm{~dB}$ at $-46^{\circ}$ for simulation and measurement.

The second point in operating impedance bandwidth of the antenna was taken at $17 \mathrm{GHz}$ which is near the center frequency. The co-polarized and cross-polarized magnitudes and phases were evaluated in simulation and verified in measurement for both E-plane and H-plane. As per the recorded data, the maximum magnitude and phase of the Co-pol E-plane at $17 \mathrm{GHz}$ was $8.28 \mathrm{~dB}$ and $9.34 \mathrm{~dB}$ both at $0^{\circ}$ for simulation and measured result. Likewise, the X-pol magnitude and phase values were found to be $-11.7 \mathrm{~dB}$ at $-2^{\circ}$ and $-5.84 \mathrm{~dB}$ at $45^{\circ}$ as shown in Figure 7(c) for E-plane at $17 \mathrm{GHz}$. Similarly, the Co-pol and X-pol magnitude of the H-plane at $17 \mathrm{GHz}$ is shown in Figure 7(d), and the maximum values for simulated and measured Co-pol were $7.46 \mathrm{~dB}$ and $7.24 \mathrm{~dB}$ both oriented at $0^{\circ}$. The $\mathrm{X}$-pol magnitude for this frequency was -4.32 at $2^{\circ}$ for simulation and $-2.94 \mathrm{~dB}$ at $-69^{\circ}$ for measurement results.

The third and last point of radiation pattern characterization was taken near the upper end of the operating range, i.e., at $21 \mathrm{GHz}$. Figure 7(e) and 7(f) show Co-pol and X-pol or E-plane and H-plane of the antenna for both simulation and measurement. The maximum main lobe magnitude and phase of the E-plane Co-pol field were observed to be $9.01 \mathrm{~dB}$ in simulation and $10.1 \mathrm{~dB}$ in measurement both are predominantly oriented at $0^{\circ}$. The X-pole values for E-plane simulation were $-4.47 \mathrm{~dB}$ which is the highest value achieved in simulation at $-49^{\circ}$ while the experimental values of $\mathrm{X}$-pol were found to be $-0.0656 \mathrm{~dB}$ at $-56^{\circ}$. Similarly, the Co-pol and X-pol magnitude and phase of $\mathrm{H}$-plane at $21 \mathrm{GHz}$ is shown in Figure 7(f).

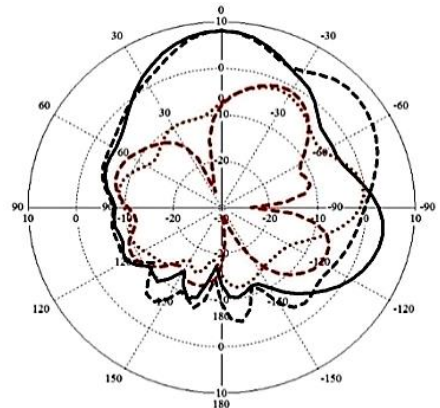

(a)

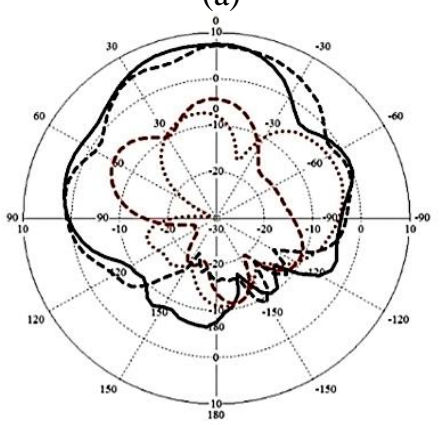

(d)

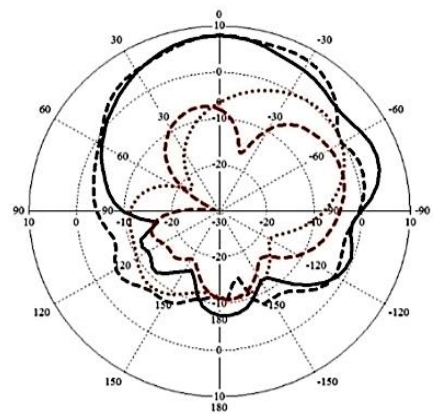

(b)

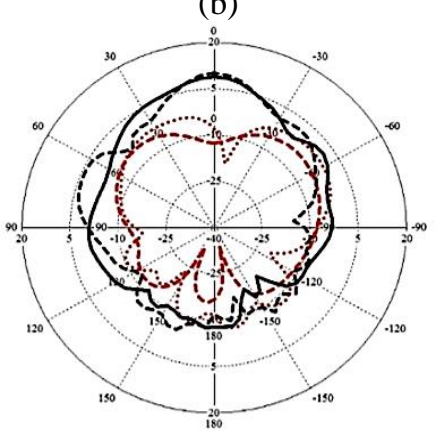

(e)

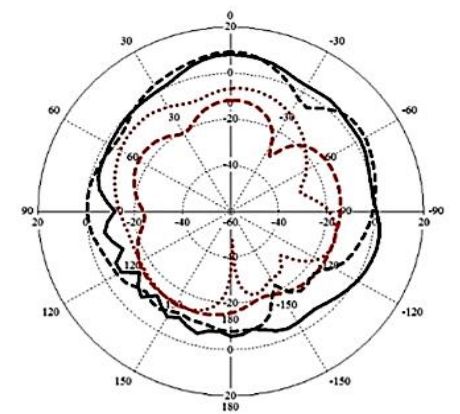

(c)

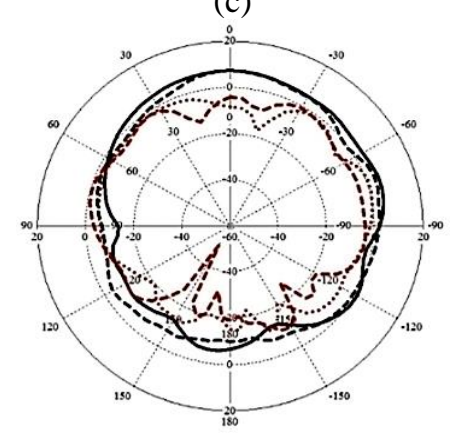

(f)

Figure 7. Simulated (- - -), measured (-) Co-polarized (black) and cross-polarized (red) radiation pattern of NSDRA, (a) E-Plane 13.5 GHz, (b) H-plane $13.5 \mathrm{GHz}$, (c) E-Plane $17 \mathrm{GHz}$, (d) H-Plane $17 \mathrm{GHz}$,

(e) E-Plane $21 \mathrm{GHz}$, (f) H-Plane $21 \mathrm{GHz}$

The maximum main lobe magnitude of Co-pol was found to be at $0^{\circ}$ for both simulation and measurement having magnitude values of $7.25 \mathrm{~dB}$ and $7.35 \mathrm{~dB}$. The X-pol magnitude of the simulation was $-0.684 \mathrm{~dB}$ oriented at $75^{\circ}$ and for measurement, this value increases slightly to $0.291 \mathrm{~dB}$ oriented at $-70^{\circ}$ and away from the maximum magnitude value of Co-pol planes.

The radiation pattern characterization results indicate that the proposed inhomogeneous nested square shape dielectric resonator was excited in the transverse electric $\left(\mathrm{TE}_{11}\right)$ mode having magnetic dipole-like radiation characteristics. The broadside radiation pattern of the antenna throughout the operating bandwidth of the antenna confirms the presence and excitation of TE mode with enhanced bandwidth due to the presence of air-gap in the resonator. Further, the magnitude and phase values of Co-pol and X-pol of the proposed inhomogeneous NSDRA suggests that this antenna can be effectively used for wideband 
application in modern communication devices requiring stable radiation characteristics and simple planer circuits. The average value of the Co-pol field was found to be approximately $7.5 \mathrm{~dB}$ oriented at $0^{\circ}$ while the average $\mathrm{X}$-pol magnitude throughout the operating bandwidth of the antenna was recorded as $-2.1 \mathrm{~dB}$ away from the angular orientation of the co-polarized main lobe.

The realized gain characterization results are presented for both simulation and experimental measurements using the 'gain standard' antenna technique. A linearly polarized gain standard horn antenna with accurately known gain value was used in the experimental characterization of the inhomogeneous nested square shape DRA. The simulated and measured gain of the proposed antenna is illustrated in Figure 8 which shows a stable gain response within the operating impedance bandwidth.

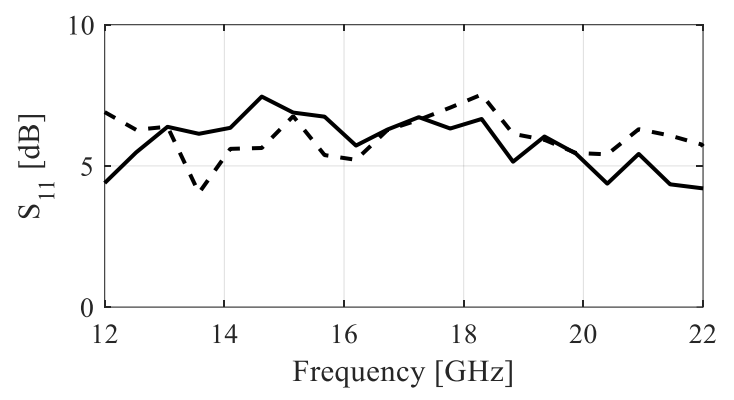

Figure 8. Simulated and measured gain of the inhomogeneous nested square DRA

As it can be seen from the graph, the simulated value of the gain is approximately $0.5 \mathrm{dBi}$ higher than that of the measured gain which is because of the imperfect prototyping, human and mechanical errors. The average gain values of both the simulation and measurement were calculated to be approximately $6.5 \mathrm{dBi}$ which is acceptable for this type of wideband antennas.

\section{CONCLUSION}

In this paper, a new nested square dielectric resonator has been investigated for wideband antenna applications in the microwave. A detailed theoretical, simulation, and experimental analyses showed that by using NSDR for antenna applications, a remarkable improvement in its performance can be achieved. The inhomogeneity (air-gap) in a solid square dielectric resonator was introduced in the $\phi$-direction so that its basic geometry and field characteristics are maintained. With this approach, the impedance bandwidth response of the antenna can be effectively enhanced. The radiation pattern characterization also showed a relatively stable response throughout the operating impedance bandwidth with a high magnitude of co-polarized fields. It can be concluded that the appropriate introduction of inhomogeneity in the solid single permittivity resonators can greatly improve the performance of DRA's without compromising their simple geometry and mechanical stability.

\section{ACKNOWLEDGEMENTS}

This work was supported by ADEK award for research excellence (AARE19-245).

\section{REFERENCES}

[1] D. Gangwar, et al., "Frequency Selective Surface as Superstrate on Wideband Dielectric Resonator Antenna for Circular Polarization and Gain Enhancement," Wireless Personal Communications, vol. 97, pp. 3149-3163, 2017.

[2] A. Petosa and A. Ittipiboon, "Dielectric Resonator Antennas: A Historical Review and the Current State of the Art," IEEE Antennas and Propagation Magazine, vol. 52, no. 5, pp. 91-116, 2010.

[3] U. Ullah, et al., "A review of wideband circularly polarized dielectric resonator antennas," China Communications, vol. 14, no. 6, pp. 65-79, 2017.

[4] O. S. Dautov, et al., "New proposed spherical slotted antenna covered by the layers of dielectric material and plasma," International Journal of Electrical and Computer Engineering (IJECE), vol. 10, no 2, pp. 1728-1735, 2020.

[5] N. F. Sallehuddin, et al., "Dielectric resonator reflectarray antenna unit cells for 5G applications," International Journal of Electrical and Computer Engineering (IJECE), vol. 8, no. 4, pp. 2531-2539, 2018. 
[6] Z. Ahmad and J. Hesselbarth, "On-chip dual-polarized dielectric resonator antenna for millimeter-wave applications," IEEE Antennas and Wireless Propagation Letters, vol. 17, no. 10, pp. 1769-1772, 2018.

[7] M. R. Nikkhah, et al., "Compact low-cost phased array of dielectric resonator antenna using parasitic elements and capacitor loading," IEEE Transactions on Antennas and Propagation, vol. 61, no. 4, pp. 2318-2321, 2013.

[8] A. Azari, et al., "A new super wideband fractal monopole-dielectric resonator antenna," IEEE Antennas and Wireless Propagation Letters, vol. 12, pp. 1014-1016, 2013.

[9] M. Abedian, et al., "Ultrawideband dielectric resonator antenna with wlan band rejection at $5.8 \mathrm{GHz}$," IEEE Antennas and Wireless Propagation Letters, vol. 12, pp. 1523-1526, 2013.

[10] A. Kiyani, et al., "A wideband circularly polarized dielectric resonator antenna over a metasurface," IEEE International Symposium on Antennas and Propagation \& USNC/URSI National Radio Science Meeting, Boston, MA, pp. 2085-2086, 2018.

[11] I. B. Mabrouk, et al., "A Novel Design of Radiation Pattern-Reconfigurable Antenna System for Millimeter-Wave 5G Applications," IEEE Transactions on Antennas and Propagation, vol. 68, no. 4, pp. 2585-2592, 2020.

[12] Y. Qian and Q. Chu, "A broadband hybrid monopole-dielectric resonator water antenna," IEEE Antennas and Wireless Propagation Letters, vol. 16, pp. 360-363, 2017.

[13] R. Zhou, et al., "Liquid-based dielectric resonator antenna and its application for measuring liquid real permittivities," Microwave Antennas Propag., vol. 8, no. 4, pp. 255-262, 2014.

[14] Y. Li and K. M. Luk, “A water dense dielectric patch antenna,” IEEE Access, vol. 3, pp. 274-280, 2015.

[15] M. Mrnka and Z. Raida, "Enhanced-gain dielectric resonator antenna based on the combination of higher-order modes," IEEE Antennas and Wireless Propagation Letters, vol. 15, pp. 710-713, 2016.

[16] M. F Ain, et al., "Bi-polarized Dual-segment Rectangular Dielectric Resonator Antenna," IETE Journal of Research, vol. 59, pp. 739-743, 2013.

[17] Z. Ahmad and J. Hesselbarth, "On-Chip Dual-Polarized Dielectric Resonator Antenna for Millimeter-Wave Applications," IEEE Antennas and Wireless Propagation Letters, vol. 17, no. 10, pp. 1769-1772, 2018.

[18] Z. Rahimian, "Circularly polarized cylindrical dielectric resonator antenna with different shapes cross slot," International Journal of Electrical and Computer Engineering (IJECE), vol. 4, no. 6, pp. 974-978, 2014.

[19] H. Younesiraad, et al., "Small multi-band rectangular dielectric resonator antennas for personal communication devices," International Journal of Electrical and Computer Engineering (IJECE), vol. 4, no. 1, pp. 1-6, 2014

[20] A. A. Kishk and W. Huang. "Size-Reduction Method for Dielectric-Resonator Antennas," IEEE Antennas and Propagation Magazine, vol. 53, pp. 26-38, 2011.

[21] A. Rashidian, et al., "On the matching of microstrip-fed dielectric resonator antennas," IEEE Transactions on Antennas and Propagation, vol. 61, pp. 5291-5296, 2013.

[22] W. Y. Feng, et al., "Bandnotched UWB rectangular dielectric resonator antenna," Electronics Letters, vol. 50, pp. 483-484, 2014.

[23] P. Rezaei, et al., "Design of wideband dielectric resonator antenna with a two segment structure," Progress in Electromagnetics Research, vol. 66, pp. 111-124, 2006.

[24] D. Kajfez and P. Guillon, "Dielectric Resonators," Norwood, MA, Artech House, Inc., pp. 1-547, 1986.

[25] U. Ullah, et al. "Design of a novel dielectric resonator antenna using MgTiO3-CoTiO3 for wideband applications," Materials \& Design, vol. 85, pp. 396-403, 2015.

[26] U. Ullah, et al., "A novel multi-permittivity cylindrical dielectric resonator antenna for wideband applications," Radioengineering, vol. 23, pp. 1071-1076, 2014. 